\title{
Impact of fatty acid-modified dairy product consumption on arterial stiffness and ambulatory blood pressure: results from the RESET study
}

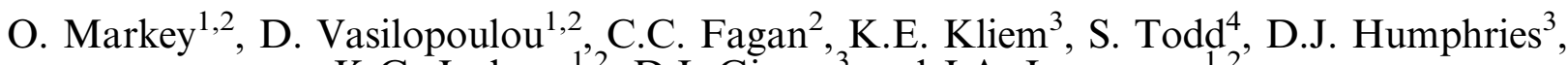 \\ K.G. Jackson ${ }^{1,2}$, D.I. Givens ${ }^{3}$ and J.A. Lovegrove ${ }^{1,2}$ \\ ${ }^{1}$ Hugh Sinclair Unit of Human Nutrition and Institute for Cardiovascular and Metabolic Research, ${ }^{2}$ Department of \\ Food and Nutritional Sciences, ${ }^{3}$ Food Production and Quality Division, University of Reading and ${ }^{4}$ Department of \\ Mathematics and Statistics, University of Reading, Reading, RG6 6 AP
}

Reduction of dietary saturated fatty acid (SFA) intake to $\leqslant 10 \%$ of total energy (TE) is a significant public health strategy for CVD risk reduction. Data from the National Diet and Nutrition Survey suggests that the UK adult population currently exceeds the target for dietary SFA intake, at $11.9 \% \mathrm{TE}^{(1)}$. As milk and dairy products (including butter) contribute to almost one third of SFA intake ${ }^{(1)}$, they are often targeted for SFA reduction. However, restricting dairy intake could lead to inadequate dietary intakes of protein and micronutrients ${ }^{(2)}$. Natural modification of the fatty acid (FA) profile of ruminant milk, through plant oil supplementation, provides an alternative strategy for lowering the population's intake of SFA and would help to minimise the movement of SFA from milk into other aspects of the food chain ${ }^{(3,4)}$. This study examined the impact of FA-modified dairy product consumption on novel CVD risk markers including arterial stiffness, as assessed by pulse wave analysis (PWA), and 24-h ambulatory blood pressure (ABP), when compared to regular, matched alternatives.

Fifty-four adults at moderate CVD risk (31 men, 23 women; age 53 (SD 13) years; BMI $25 \cdot 8$ (SD 3.4) $\mathrm{kg} / \mathrm{m}^{2}$ ) participated in the RESET (REplacement of SaturatEd fat in dairy on Total cholesterol) study, a randomised, crossover, double-blinded controlled trial (ClinicalTrials.gov: NCT02089035). An iso-energetic daily dietary exchange (41 g/d total fat) was achieved by replacing habitual dairy foods/snacks with regular or FA-modified UHT milk, Cheddar cheese and butter (FA-modified dairy products (FA composition vs. regular dairy products: Total SFA: $-7.0 \mathrm{~g} / \mathrm{d}, \mathrm{C} 18: 1$ cis: $3.0 \mathrm{~g} / \mathrm{d}, \mathrm{C} 18: 1$ trans: $2.4 \mathrm{~g} / \mathrm{d}$ ). PWA and 24-h ABP were measured at baseline and following each 12-week intervention period. PWA was measured in the fasted state and used to calculate augmentation index, corrected for a heart rate of 75 beats/min (AIx). 24-h ABP was monitored in the home environment $>24-\mathrm{h}$ before clinical visits. Daytime and night-time ABP measurements were estimated based on participants' sleep and wake time records. Pulse pressure (PP) was calculated as the difference between systolic blood pressure (SBP) and diastolic blood pressure (DBP) values. A mixed model analysis, adjusted for baseline values, gender, age and BMI, was used to assess differences between the two intervention periods.

Preliminary analyses revealed no significant treatment or period effects for AIx $(P=0.48)$ or SBP, DBP or PP during daytime, night-time and over a 24-h period (all $P>0.05$ ). When incorporated into the diet for a 12 -week period, these data indicate that the intake of regular and FA-modified dairy products did not have differential effects on arterial stiffness or ABP parameters.

These preliminary findings support evidence, which suggest a neutral effect of milk consumption on markers of cardiometabolic health $^{(5)}$. Future results will reveal the impact of FA-modified dairy consumption on other novel CVD risk indicators including postprandial lipid metabolism, inflammatory markers and endothelial function.

This research was supported by the Medical Research Council (MR/K020218/1), with food in-kind from Arla Foods UK and AAK (UK) Ltd.

1. Bates et al. (2014) National Diet and Nutrition Survey: Headline results from Years 1 and 4 combined of the rolling programme 2008/9-2011/12.

2. Markey et al. (2014) Nutr Bull 39, 161-171.

3. Kliem and Givens (2011) Annu Rev Food Sci, Technol. 2, 21-36.

4. Markey et al. (2015) Proc Nutr Soc 74, OCE1: E52.

5. Lamarche et al. (2016) Can J Cardiol. doi:10.1016/j.cjca.2015.12.033 